\title{
Injury Proneness and Factors of Physiology and Psychology Among College Freshmen: A Matched Case-Control Study
}

\author{
Jing ZENG ${ }^{1, a}$, Bing XIANG ${ }^{1, b^{*}}$, Yan-Pei DING ${ }^{1, c}, X_{1}$ TAO $^{1, d}$, Chang-Cai ZHU ${ }^{1, e}$, \\ and Guan-Min CHEN ${ }^{2, f}$ \\ ${ }^{1}$ School of Public Health, Wuhan University of Science and Technology, Wuhan, Hubei, China. \\ ${ }^{2}$ Department of Community Health Sciences, University of Calgary, Calgary, Alberta, Canada.

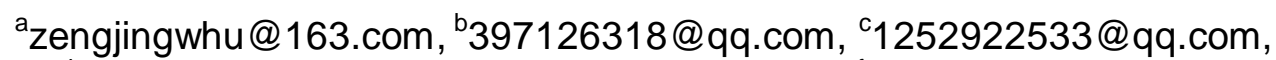 \\ d949705963@qq.com, ${ }^{\mathrm{e}} 794421615 @ q q . c o m,{ }^{\dagger}$ guchen@ucalgary.ca \\ ${ }^{*}$ Corresponding author
}

Keywords: injury; freshmen; physiological and psychological factors; case-control study

\begin{abstract}
Objective: The aim of this research was to investigate the relationships of some physiological and psychological factors with one time injury or more than one time injuries among college freshmen students. Methods: 80 injured students (one time injury group) and 80 matched controls, 16 injured students (more than one time injuries group) and 64 matched controls are recruited among 1282 freshmen from two schools, in a university in Wuhan, China. The data about injury and psychological and physiological factors were collected from 1 September 2011 to 31 August 2012. In terms of statistical analysis, chi-square tests as well as conditional logistic regression analyses were applied and odds ratios (OR) were determined.Results: Over the study year, $6.2 \%$ of the students had a one time injury, and $1.3 \%$ of them had more than one time injuries. The one time injury was significantly related to higher total score of health risk behavior (HRB) [adjusted odds ratio (aOR) 1.1, 95\% confidence interval (95\%CI 1.0 1.3)], poor corrected vision (aOR 2.1, 95\%CI 1.0 4.2), and time on the target with the noise interference (aOR 0.8, 95\% CI 0.7 0.9). While, the occurrence of more than one time injuries was related to have addictive behavior (aOR 2.2, 95\% CI 1.1 4.3), type A behavior personality (aOR 6.3, 95\%CI 1.3 31.4), and longer total reaction time (aOR 2.6, 95\%CI 1.1 6.2). Conclusion: It was concluded that physiological and psychological factors were significantly associated with the occurrence of injuries among college freshmen, and the factors were different between the one time injury group and more than one time injuries group.
\end{abstract}

\section{Introduction}

Injuries are very common among adolescents and young adults, and have serious health and socio-economic consequences such as disability, hospitalization, and school absence [1-5]. In China, the freshmen just complete their competing admission examination in the last high school year and moved into new environment in university. Compared with high school, the students have more entertainment time for physical activities. While the students left their home, and have more physiological and psychological burdens than high school students. Previous students indicated the undergraduates in the first school year are more likely to be injured than other undergraduates [6-7].

Studies showed injuries among students are associated with factors of age, sex, physical ability, lack of knowledge, personality, behavior, and environment characteristics [1-3, 8-10]. Boles et al. reported that individuals with injures had a higher detection rate of outgoing personality and anxiety [11]. Studies from Marcelli et al. showed that repeated accidents are more likely to occur in adolescents with depression, anxiety and impulsive characters [7]. Among Chinese undergraduates, Shi et al. reported that unintentional injuries have a positive association with type A behavior pattern, especially the hostility component [12]. Based on the patients from emergency departments, the types of injuries [2, 4, 13-15], sociodemographic, family and environment factors are widely investigated among 
undergraduates [1, 8-10]. Few studies has been performed to explore the association of personality, psychological, physiological, and behavior factors with the injuries among first year undergraduates. This case-control study investigated the psychological and physiological factors, including health risk behavior, visual acuity, focus capability, response capability, mental health status, and self-reported personality and injury proneness among freshmen from a university, Wuhan, China.

\section{Methods}

\section{Research design and study population}

A total of 80 injured students and 80 matched controls are recruited among 1282 freshmen from two schools, in Wuhan University of Science and Technology, Wuhan, China. The data of injury were collected from 1 September 2011 to 31 August 2012. There are 16 students with more than one time injuries during this study period. We also selected them as case group for more than one time injuries, and matched as 1:4 controls. A total of 64 students were selected as control group for 16 students with more than one time injuries. Based on the research protocol, the case and control was matched as same sex, same age, and from same hometown.

The injured students were interviewed using self-administered questionnaire in a week after injury occurred. The matched controls were selected from same school and were interviewed using the same questionnaire. The study was approved by the internal review board of Medical School, Wuhan University of Science and Technology. The study was conducted in Wuhan, the capital of Hubei province, and was known for having many universities. Nine graduate students from major public health were trained as interviewers and performed the interview.

\section{Diagnosis of injuries}

An injury was diagnosed which met any one of the following two situations: (1) the student was diagnosed to have an injury by a doctor at a hospital or clinic; or (2) the student was absent from school or rested for a day or longer because of injury[16]. Injuries were classified into eight major types according to the International Classification of Diseases $10^{\text {th }}$ Revision (ICD-10), including transport accidents, poisoning, falls, asphyxia, burns, pet bites, mechanical injuries and electric injuries.

\section{Study variables}

The students were interviewed using the questionnaire, which consists of following parts. 1) sociodemographic factors which includes variables of age, gender, parents education etc.; 2) the injuries, frequency, times, types, and locations of injuries, time; The 3)Health risk behavior (HRB) measurement; 4)psychological health status measurement; and 5)type A personality measurement.

HRB measurement: HRB was measured using The Youth Risk Behavior Surveillance System (YRBSS) which developed by U.S. Centers for Disease Control and Prevention. The translated Chinese version was revised for adopting undergraduates' characteristics and cultures. It consists of the following 7 domains with 24 items: (a) behavior cause unintentional injuries; (b) behavior cause intentional injuries; (c) substance abuse like tobacco, alcohol, etc.; (d) addictive behavior; (e) sexual behavior; (f) unhealthy eating behavior; (g) lack of physical activity. Each item was rated on a four-point scale: never $=1$, rarely $=2$, sometimes $=3$, and often $=4$. The higher the score means the higher the degree of risk of the behavior.

Psychological health measurement: The Symptom Checklist -90 ( SCL-90), a 90-item self- report instrument was used to measure the psychological health. SCL-90, that has been designed to evaluate a broad range of psychological problems and symptoms, and has been used to measure the mental health status among students [17]. A SCL-90 Chinese version has been translated, and has been widely used to measure the psychological factors among Chinese undergraduates. A total score $>160$ points or positive items over 43 or any factor score more than 2 points was indicative of malfunctioning according to the results of the national norm. 
Type A personality measurement: The type A personality was measured using the Type A Behavior Pattern (TABP) scale developed by Friedman and Rosenman. The TABP Chinese version was translated and revised by the Chinese National Collaborative Study Group [18]. There are three dimensions: TU (time urgency), $\mathrm{CH}$ (competitive hostility), and L(lie) with a total of 60 items in the scale. If the score for the $\mathrm{L}$ dimension was higher than 7 , the measurement was invalid and would be deleted in the final data analysis. The total score for the students was summed up for each dimensions in the scale. According to the mean $($ mean $=27)$ and standard deviation $(\mathrm{SD}=8)$ of the total score of the scale, we grouped the total score as five groups: $\mathrm{A}(\geq 36), \mathrm{mA}(28-35), \mathrm{M}(27), \mathrm{mB}(19-26)$, and $\mathrm{B}(\leq 18)[18]$. The students with Groups A and mA categorized as type A, and the others as not type A. [19].

\section{Laboratory test}

Eyesight test: The ' $E$ ' international standard logarithmic visual acuity chart was used to measure the visual acuity. The health professional staff in the university hospital performed this test for both case and control students. both naked vision and the vision with glasses for the students with impaired vision were measured. Student with binocular (or monocular) uncorrected or corrected visual acuity $<1.0$ should be considered poor vision.

Focus test: A Focus Tester (EP701C, Shanghai, China) was used to measure the focus capability. The focus test was performed in under both quiet environment and a noise environment, respectively by health professional staffs. The time on the target and the frequency of off-target with or without noise were measured for each student.

Reaction time \& motion time test: A Reaction time \& motion time tester (EP206-P, Shanghai, China) was used to measures the reaction time and motion time when students respond to a target stimulus. A total of 20 times tests were performed for each student. The total reaction time, the total motion time, and the number of errors for each student were summed up for analysis.

\section{Statistical analysis}

Statistical analysis was performed using IBM SPSS statistics version 17.0 (IBM corp. Armonk, NY). Matched paired $t$ test and Mcnemar chi-square test were used to test differences of risk factors between case and control. The crude odds ratios (OR), and adjusted odds ratios(aOR) with $95 \%$ confidence intervals (CI) for one time injuries and more than one times injuries were estimated using conditional logistic regression for each factor. For multivariate conditional logistic regression model, the step-wise method was used to select the factors associated with the injuries, and the factors were selected to enter the model for alpha $=0.05$, and it was excluded from the model for alpha $=0.1$.

\section{Results}

A total of $96(7.5 \%)$ injuries were observed among the 1282 participants during the study period. Among of them, $80(6.2 \%)$ students have one time injuries, and $16(1.3 \%)$ students have more than one injury. For matched case and control students, after the TABP test, 3 paired students for one time injury, and 2 paired students for more than one time injuries were excluded due to the score of the $\mathrm{L}$ dimension was higher than 7 . Therefore, a total of 77 pairs and 14 blocks were analyzed in this study.

HRB, SCL-90, Focus test, et al. between injured and uninjured students Table 1 showed that the occurrence of one time injury was significantly related to the total scores of HRB, time on the target with the noise, and poor corrected vision. Whereas more than one time injuries were associated with behavior cause intentional injuries, substance abuse, addictive behavior, the total scores of HRB, the times on the target with the noise, total reaction time, total motion time, and type A personality.

Risk factors associated with one time injuries For one time injuries, The students with higher total score of HRB (aOR 1.1, 95\%CI 1.0 1.3), poor corrected vision (aOR 2.1, 95\%CI 1.0 4.2), and time on the target with the noise interference (aOR 0.8, 95\% CI 0.7 0.9) are more likely to be injured.(Table2) . 
Table 1 Physiological and psychological factors of these freshmen students in cases and controls

\begin{tabular}{|c|c|c|c|c|c|c|}
\hline \multirow{3}{*}{ Variables } & \multicolumn{2}{|c|}{ One time injury } & & \multicolumn{2}{|c|}{$\begin{array}{l}\text { More than one time } \\
\text { injuries }^{\mathrm{a}}\end{array}$} & \multirow[b]{3}{*}{$P$} \\
\hline & $\begin{array}{c}\text { Cases } \\
(\mathrm{N}=77)\end{array}$ & $\begin{array}{c}\text { Controls } \\
(\mathrm{N}=77)\end{array}$ & & $\begin{array}{c}\text { Cases } \\
(\mathrm{N}=14)\end{array}$ & $\begin{array}{c}\text { Controls } \\
(\mathrm{N}=56)\end{array}$ & \\
\hline & $M \pm S D$ & $M \pm S D$ & $P$ & $M \pm S D$ & $M \pm S D$ & \\
\hline \multicolumn{7}{|l|}{ Health risk behaviors } \\
\hline Behavior cause unintentional injuries & $5.4 \pm 1.4$ & $5.2 \pm 1.3$ & 0.205 & $5.2 \pm 1.0$ & $5.2 \pm 1.1$ & 0.753 \\
\hline Behavior cause intentional injuries & $6.5 \pm 1.3$ & $6.3 \pm 1.1$ & 0.525 & $7.4 \pm 2.4$ & $6.2 \pm 1.1$ & $0.048^{*}$ \\
\hline Substance abuse & $7.8 \pm 1.6$ & $7.4 \pm 1.5$ & 0.058 & $8.5 \pm 1.8$ & $7.6 \pm 1.4$ & $0.028^{*}$ \\
\hline Addictive behavior & $3.5 \pm 1.2$ & $3.3 \pm 1.0$ & 0.238 & $4.0 \pm 1.1$ & $3.2 \pm 1.1$ & $0.048^{*}$ \\
\hline Sexual behavior & $1.1 \pm 0.4$ & $1.0 \pm 0.2$ & 0.439 & $1.0 \pm 0.0$ & $1.1 \pm 0.5$ & 0.423 \\
\hline Unhealthy eating behavior & $3.3 \pm 0.9$ & $3.1 \pm 0.8$ & 0.076 & $3.5 \pm 1.3$ & $3.1 \pm 0.9$ & 0.150 \\
\hline Lack of physical activity & $5.9 \pm 1.5$ & $5.5 \pm 1.4$ & 0.082 & $6.1 \pm 1.8$ & $5.7 \pm 1.7$ & 0.478 \\
\hline Total score of health risk behavior & $31.3 \pm 4.3$ & $29.7 \pm 3.6$ & $0.015^{*}$ & $33.3 \pm 5.4$ & $30.0 \pm 3.5$ & $0.018^{*}$ \\
\hline \multicolumn{7}{|l|}{ Focus test } \\
\hline Time on the target without the noise (s) & $55.1 \pm 3.6$ & $55.9 \pm 3.6$ & 0.128 & $53.3 \pm 3.2$ & $55.9 \pm 5.7$ & 0.085 \\
\hline Frequency of off-target without the noise & $16.7 \pm 14.0$ & $17.7 \pm 12.2$ & 0.582 & $31.2 \pm 12.9$ & $24.7 \pm 14.0$ & 0.109 \\
\hline Time on the target with the noise (s) & $52.5 \pm 3.4$ & $54.1 \pm 2.7$ & $0.003^{* *}$ & $50.0 \pm 2.6$ & $53.2 \pm 4.5$ & $0.042^{*}$ \\
\hline Frequency of off-target with the noise & $19.2 \pm 14.7$ & $20.7 \pm 13.0$ & 0.381 & $23.7 \pm 12.2$ & $19.9 \pm 13.8$ & 0.303 \\
\hline \multicolumn{7}{|l|}{ Reaction time \& motion time test } \\
\hline Total reaction time (s) & $5.8 \pm 1.13$ & $5.8 \pm 1.0$ & 0.379 & $6.2 \pm 0.8$ & $5.5 \pm 1.0$ & $0.037^{*}$ \\
\hline Total motion time (s) & $6.1 \pm 0.6$ & $6.0 \pm 0.7$ & 0.717 & $6.4 \pm 0.6$ & $5.9 \pm 0.7$ & $0.034^{*}$ \\
\hline \multirow[t]{2}{*}{ Frequency of errors } & $0.2 \pm 0.5$ & $0.2 \pm 0.5$ & 1.000 & $0.1 \pm 0.4$ & $0.4 \pm 0.6$ & 0.211 \\
\hline & $n(\%)$ & $n(\%)$ & $P$ & $n(\%)$ & $n(\%)$ & $P$ \\
\hline \multicolumn{7}{|l|}{ Eyesight test } \\
\hline Poor naked vision & $67(79.2)$ & $69(89.6)$ & 0.617 & 13(92.9) & $51(91.1)$ & 0.831 \\
\hline Poor corrected vision & $44(57.1)$ & $27(35.1)$ & $0.006^{* *}$ & $8(57.1)$ & $17(30.4)$ & 0.061 \\
\hline psychological problems(SCL-90) & $21(27.3)$ & $25(32.5)$ & 0.480 & $5(35.7)$ & $17(30.4)$ & 0.708 \\
\hline Type A personality & $21(27.3)$ & $23(29.9)$ & 0.724 & $7(50.0)$ & 11(19.6) & $0.038^{*}$ \\
\hline
\end{tabular}

Notes: More than one time injuries indicates have many times $(\geq 2)$ injuries during the study year. $* P<0.05$,

$* * P<0.01$ (two-tailed)

Table 2 Univariate and multivariate conditional-logistic regression results of college students with one time injury or more than one time injuries and controls

\begin{tabular}{|c|c|c|c|c|c|c|c|c|}
\hline \multirow{2}{*}{ Factors } & \multicolumn{4}{|c|}{ Univariate analysis $^{\mathrm{a}}$} & \multicolumn{4}{|c|}{ Multivariate analysis ${ }^{b}$} \\
\hline & $O R^{\mathrm{c}}$ & \multicolumn{2}{|c|}{$95 \% C I$} & $P$ & $a O R^{\mathrm{c}}$ & \multicolumn{2}{|c|}{$95 \% C I$} & $P$ \\
\hline \multicolumn{9}{|l|}{ one time injury } \\
\hline Total score of health risk behavior & 1.1 & 1.0 & 1.2 & $0.015^{*}$ & 1.1 & 1.0 & 1.3 & $0.029^{*}$ \\
\hline Poor corrected vision & 2.3 & 1.2 & 4.4 & $0.012^{*}$ & 2.1 & 1.0 & 4.2 & $0.041^{*}$ \\
\hline Time on the target with the noise interference & 0.8 & 0.7 & 0.9 & $0.006^{* *}$ & 0.8 & 0.7 & 0.9 & $0.022^{*}$ \\
\hline \multicolumn{9}{|l|}{ more than one time injuries } \\
\hline Behavior cause intentional injuries & 1.6 & 1.0 & 2.5 & $0.048^{*}$ & 0.8 & 0.4 & 1.8 & 0.638 \\
\hline Substance abuse & 1.8 & 1.1 & 2.9 & $0.028^{*}$ & 1.6 & 0.7 & 3.6 & 0.221 \\
\hline Addictive behavior & 1.7 & 1.0 & 3.0 & $0.048^{*}$ & 2.2 & 1.1 & 4.3 & $0.029^{*}$ \\
\hline Total score of health risk behavior & 1.2 & 1.0 & 1.4 & $0.018^{*}$ & 0.8 & 0.5 & 1.5 & 0.562 \\
\hline Type A personality & 3.5 & 1.1 & 11.4 & $0.038^{*}$ & 6.3 & 1.3 & 31.4 & $0.024^{*}$ \\
\hline Time on the target with the noise interference & 0.9 & 0.8 & 0.9 & $0.042^{*}$ & 0.8 & 0.6 & 1.1 & 0.180 \\
\hline Total reaction time & 2.0 & 1.0 & 3.7 & $0.034^{*}$ & 2.6 & 1.1 & 6.2 & $0.025^{*}$ \\
\hline Total motion time & 2.7 & 1.1 & 7.1 & $0.037^{*}$ & 1.3 & 0.3 & 5.6 & 0.674 \\
\hline
\end{tabular}

Notes: ${ }^{\mathrm{a}}$ Estimated from logistic regression model including each single factor separately. ${ }^{\mathrm{b}}$ Estimated from logistic

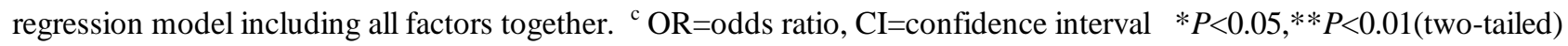

Risk factors associated with more than one time injuries The factors of behavior cause intentional injuries, substance abuse, addictive behavior, total score of health risk behavior, time on the target with the noise, total reaction time, total motion time, and type A personality are associated with 
more than one time injuries among univariates conditional logistic regression models. For multivariate analysis, the students with addictive behavior (aOR 2.2, 95\%CI 1.1 4.3), type A behavior personality (aOR 6.3, 95\% CI 1.3 31.4), and longer total reaction time (aOR 2.6, 95\% CI 1.1 6.2) are more likely to be injured. The aOR of injuries among freshmen with type A behavior personality was strongly higher than that among freshmen with other type behavior personality.

\section{Discussion}

In this matched-pair case-control study, we found that students' psychological and physiological characteristics were significantly associated with the occurrence of injury among freshmen students. We also found the factors associated the one time injury group and more than one time injuries group were different. The one time injury was related to higher total score of HRB, poor corrected vision, and time on the target with the noise interference. While, the occurrence of more than one time injuries was related to have addictive behavior, type A behavior personality, and longer total reaction time.

The present study found that in comparison with controls, those who susceptible to a one time injury were associated with a higher total score of HRB, and those who susceptible to more than one time injuries were associated with addictive behavior. It is well known that the proportion of students experience behavior disorders is increased significantly. In the US, Weller et al. evaluated HRB in students aged 14-20 and reported that 35.9\% showed signs of health risk behavior pattern [20]. Yang et al. reported that $56.1 \%$ college students have health risk behaviors in Jiangxi province in China, most of these behaviors can cause injuries directly or indirectly [21]. The addictive behavior in our study included internet addictive and gambling. A study in China reported that the internet addiction rate was $9.8 \%$ in college students, and phenomena of staying up late for playing games or chatting on the internet were very common among college students [22]. Health promotion within schools should be introduced to improve students' self-control ability, and to provide a good environment of learning and entertainment for the promotion of safer, supportive school environments.

This study found that poor corrected vision was a risk factor for one time injury when adjusting for all factors considered. Some studies reported that poor naked vision was related to injury in school students [23-24], but in the present study, the difference of poor naked vision rate between cases and controls was not statistically significant, however, the poor corrected vision was associated. This may be explained by the high rate of poor naked vision in freshmen in our study, and most of them wearing glasses. This study also found that these students did not check their vision and replace their unsuitable glasses regularly, for the rates of poor corrected vision were very high (more than 30\%) among two group students. So health education work such as enhance their awareness of eye protection, and let them to take regular visual activity examination was very important in injury prevention.

In this stduy, a protect factor for one time injury was a longer time on the target with the noise interference. It means that under the surrounding interference, the better the focus of attention, the smaller the risk of injury. Other studies also indicated that the training of students' attention quality is an important aspect in reducing the injuries among students. The reason may be the students have good attention, and can quickly react in hazardous environment to avoide the occurrence of injury.

Type A behavior pattern (TABP), characterized by time urgency, impatience, and hostility, has been reported to be associated with heart disease traditionally. Since 1980s, there are studies concerning the relationship between TABP and injuries, but the results are inconsistent. Several epidemiological studies have provided evidence of an association between type A personality and injuries [12, 25-26]. Among of them, one study demonstrated that runners with high scores on the type A behavior experienced significantly more injuries, especially more than one time injuries [26]. But other studies did not find any significant relationship between individual personality and injuries [27-28]. Our results added further support to the notion that students with TABP are more likely to have more than one time injuries. Compared with the non-typa A individuals, the aOR for more than one time injuries was 6.3 (95\%CI 1.3 31.4) among type A subjects.

This study also found that frequent injured subjects show significant increases in reaction time to 
stimuli compared to controls. The reaction time is the time span from the body accepting the stimuli to make the response. Those subjects who need a long time to make response always escape slowly when emergency issues occur. As reported in previous study, car drivers who caused road traffic accidents have longer reaction time [29]. Decreasing the reaction time seems to be important to avoid the injuries. Some special training can be used to improve hand-eye coordination and the synchronizing of various parts of the body, and thereby reducing the reaction time.

The small sample size of this study, especially the more than one time injuries group, certainly represented a limitation for the result extension. Second, as with any case-control study, recall bias may be possible among cases and controls. Additionally, as this is a retrospective study, it is not possible to determine a specific causality relationship between some factors and injury occurrence.

In conclusion, this study identified a number of potential risk factors for single and more than one time injuries in college freshmen students. Youth is a good time to learn to control the risks of injuries, and schools can teach students how to promote safety and prevent injuries. Interventions targeting specifically on the risk factors may help prevent and reduce the occurrence of injuries in college freshmen students in China.

\section{Acknowledgement}

This research was financially supported by the general project of Ministry of Education, Humanities and Social Science Research (Grant No.13YJAZH104).

\section{References}

[1]. Zhou L, Chen D, Dong G. Characteristics and related factors of nonfatal injuries among adolescents and college students in Shenzhen City of China. BMC Public Health. 26(2013):392

[2]. Ramirez M, Peek-Asa C, Kraus JF. Disability and risk of school related injury. Inj Prev. 10(2004):21-26

[3]. Sosnowska S, Kostka T. Epidemiology of school accidents during a six school-year period in one region in Poland. Eur J Epidemio. 19(2003):977-982

[4].Willer B, Dumas J, Hutson A, Leddy J. A population based investigation of head injuries and symptoms of concussion of children and adolescents in school. Inj Prev.10(2004):144-148

[5].Scheidt PC, Harel Y, Trumble AC, et al. The epidemiology of nonfatal injuries among US children and youth. Am J Public Health. 85(1995):932-938

[6]. Luo Y, Tao FB, Zhang AH, et al. A prospective study on the current status of repeated injuries and its risk factors in middle school and college students. Zhonghua Liu Xing Bing Xue Za Zhi. 31(2010): $30-33$

[7]. D.Marcelli., M.-A.Kasolter-pere., P.Ingrand., Proposal for an evaluation scale of the risk of repeated accidents among teenagers [J]. Annales Medico Psychologiques.166(2008): 691-701.

[8]. Shi H, Yang X, Huang C, et al. Status and risk factors of unintentional injuries among Chinese undergraduates: a cross-sectional study. BMC Public Health.11(2011):531

[9]. Mattila V, Parkkari J, Kannus P, et al. Occurrence and risk factors of unintentional injuries among 12- to 18- year old Finns- a survey of 8219 adolescents. Eur J Epidemilo.19(2004):437-444

[10]. Jia C, Zhao Z, Bo Q, et al. Incidence rates and risk factors for injury events in high school students in Lijin County, China. J Safety Res. 36(2005):133-137

[11]. Boles RE, Robe,s MC, BrownKJ, et a1. Children'S risktaking behaviors: the role of child-based perceptions of vulnerability and temperament. Pedia Psychol. 30(2005): 562-570.

[12]. Shi H, Yang X, Wang J, et al. Type A personality, hostility, time urgency and unintentional 
injuries among Chinese undergraduates: a matched case-control study. BMC Public Health. 13(2013):1066

[13].Korcha RA, Cherpitel CJ, Ye Y, et al. Alcohol use and injury severity among emergency department patients in six countries. J Addict Nurs. 24(2013):158-165

[14]. Lee CA, Choi SC, Jung KY, et al. Characteristics of patients who visit the emergency department with self-inflicted injury. J Korean Med Sci. 27(2012):307-312.

[15]. Nordqvis C, Holmqvist M, Nilsen P, et al. Usual drinking patterns and non-fatal injury among patients seeking emergency care. Public Health. 120(2006): 1064-1073.

[16]. The Society for Injury Prevention and Control of Chinese Preventive Medicine Association. Decision on criteria for defining of injury. Chin J Dis Control Pre. 15(2011):9.

[17]. L.R.Derogatis, SCL-90-R. Symptom Chechlist-90-R, Giunti OS,2011

[18]. Zhang BY. The National Collaborative Study Group for TABP \& CHD: Psychophysiological Reaction in Cardiovascular Disease. Acta Psychologica Sinica. 3(1985):314-321

[19]. Zhang SJ, Zhou QQ, Zhang RL, et al. Studies on relations between a type behavior and the infection of choleithiasis. Chin J Behav Med Sci.14(2005):217-219

[20]. Weller NF, Tortolero SR, Kelder SH, et al. Health risk behaviors of Texas students attending dropout prevention/recovery schools in 1997. J Sch Health. 69(1999):22-8.

[21]. Yang Y. Risk behavior and its influencing factors in college students in Jiangxi Province. Chin J Sch Health. 34(2013):1503-1506

[22]. Dai J, Wang S, Wang C, et al. Logistic regression analysis on risk behaviors and influencing factors among college students. Chin J Dis Control Prev. 14(2010):953-955.

[23]. Xu S, Xiang B, Xiong C, et al. Relationships between injury and visual acuity, attention, and psychologic status in undergraduate students. Chin J Public Health. 27(2011):1391-1392

[24]. Visser E, Pijl YS, Stolk PP, et a1. Accident proneness, does it exist? A review and meta-analysis [J].Accident Analysis and Prevention. 39(2007):556-564.

[25]. Wang XM, Yang L, Zhang HY, et al. Association of type-A behavior and personality with accident proneness among drivers. Chin J Public Health. 28(2012):282-284

[26]. Fields KB, Delaney M, Hinkle JS. A prospective study of type A behavior and running injuries. J Fam Pract. 30(1990):425-429

[27]. Gregg RL, Banderet LE, Reynolds KL. Psychological factors that influence traumatic injury occurrence and physical performance. Work. 18(2002):133-139

[28]. Li YZ, Wang ZG, Yin ZY, et al. The relationship of road accidents with motorcyclists' riding behavior, personality and attitudes towards safety. Psychol Sci. 31(2008):487-489

[29]. Feenstra H, Ruiter RA, Kok G. Go fast! Reaction time differences between adults and adolescents in evaluating risky traffic situations. J Health Psychol. 17(2012): 343-349. 